\title{
Tránsito hepatotorácico, complicación evolutiva de la hidatidosis hepática. Características clínicas y morbilidad de una serie prospectiva de pacientes intervenidos quirúrgicamente*
}

\author{
Drs. CARLOS MANTEROLA D. ${ }^{1,2}$, NICOLE ÁVILA A. ${ }^{1}$, JAVIERA SECO V. ${ }^{1}$, \\ PEDRO ULLOA M. ${ }^{1}$, JAVIER MORAGA C. ${ }^{1}$ GRUPO MINCIR \\ 1 Departamento de Cirugía y Traumatología. \\ 2 CIGES (Capacitación, Investigación y Gestión para la Salud Basada en Evidencia). \\ Universidad de La Frontera, Temuco, Chile.
}

\begin{abstract}
Hepatothoracic transit, evolutionary complications of hepatic echinoccocosis

Introduction: Hepatothoracic transit (HTT) is one of the evolutionary complications of hepatic echinoccocosis (HH). The aim of this study is to describe clinical characteristics and morbidity of a series of patients with HTT surgically treated. Material and Methods: Prospective series of cases. Patients operated by HTT between 1997 and 2007 in Regional Hospital of Temuco, Chile, with a minimum follow-up of 12 months and clinical controls at months 1, 6, 12, 24, 36, 48 and 60. End point was "development of morbidity". Descriptive statistic was used, applying central tendency and dispersion measured. In addition confidence intervals of $95 \%$ was calculated. Results: The series is composed by 23 patients with HTT with a median age of 48 years (16 to 75 years) and 56\% femenine. Median cyst diameter was $20 \mathrm{~cm}(8$ to $30 \mathrm{~cm}) 78.3 \%$ of the lesions were located in the right lobe of the liver. The most frequent surgical technique used was subtotal cystectomy (78.3\%); residual cavity were treated preferably by capitonage (30.4\%) or epiploplasty (34.8\%). With a median follow-up of 71 months (12 to 122 months), one verified that no patient required surgical reintervención; mortality rate was $4.3 \%$ (one patient) and registered morbidity was $26.0 \%$ (6 patients). Conclusion: HTT is associated to considerable values of post-operative morbidity and mortality.
\end{abstract}

Key words: Hydatidosis, liver hydatidosis, hydatid hepatic cyst, hepatothpracic transit, morbidity.

\section{Resumen}

Introducción: Una de las complicaciones evolutivas de la hidatidosis hepática $(\mathrm{HH})$ es el tránsito hepatotorácico (THT). El objetivo de este estudio es describir características clínicas y morbilidad de una serie de pacientes con THT intervenidos quirúrgicamente. Material y Método: Serie de casos prospectiva. Pacientes intervenidos por THT entre 1997 y 2007 en el Hospital Regional de Temuco, Chile, con seguimiento mínimo de 12 meses y controles clínicos en los meses 1, 6, 12, 24, 36, 48 y 60. La variable resultado

*Recibido el 10 de Marzo de 2009 y aceptado para publicación el 15 de Abril de 2009.

Correspondencia: Dr. Carlos Manterola D.

Casilla 54-D, Temuco, Chile. Fax: 45-325761

E-mail: cmantero@ufro.cl 
fue "desarrollo de morbilidad". Se utilizó estadística descriptiva, aplicando medidas de tendencia central y dispersión e intervalos de confianza del 95\%. Resultados: La serie está compuesta por 23 pacientes con THT, con una mediana de edad 48 años ( 16 a 75 años) y 56\% de género femenino. El diámetro quístico tuvo una mediana de $20 \mathrm{~cm}$ ( 8 a $30 \mathrm{~cm}$ ), y el 78,3\% de ellos se localizó en el lóbulo derecho del hígado. La técnica quirúrgica utilizada con mayor frecuencia fue quistectomía subtotal $(78,3 \%)$; la cavidad residual se trató preferentemente mediante capitonaje $(30,4 \%)$ o epiploplastía $(34,8 \%)$. Con una mediana de seguimiento de 71 meses (12 a 122 meses), se verificó que: ningún paciente requirió de reintervención quirúrgica; se registró una morbilidad de 26,0\% (6 pacientes) y una mortalidad de 4,3\% (un paciente). Conclusión: El THT es una entidad asociada a morbilidad y mortalidad postoperatoria relevante.

Palabras clave: Hidatidosis, hidatidosis hepática, quiste hidatídico hepático, tránsito hepatotorácico, morbilidad.

\section{Introducción}

Una de las complicaciones evolutivas de la hidatidosis hepática $(\mathrm{HH})$ es el tránsito hepatotorácico (THT); y que corresponde al compromiso simultáneo de hígado, diafragma y pulmón secundario a la migración de un quiste hidatídico hepático; cuya frecuencia en la literatura se estima entre $2 \%$ y un $11 \%$ de las series de pacientes con $\mathrm{HH}^{1}$.

Esta, es una entidad compleja de tratar debido entre otras cosas, a que no es infrecuente que estas lesiones se encuentren infectadas, generando además un absceso secundario ${ }^{2}$; o que se encuentren directamente comunicadas al árbol bronquial, hecho que se manifiesta clínicamente como tos, disnea, dolor torácico y eventualmente como biliptisis; e incluso que se encuentren comunicados a la vía biliar, produciendo colangiohidatidosis ${ }^{3}$. Todas ellas, situaciones que pueden incrementar aún más el riesgo de morbilidad postoperatoria ${ }^{4}$. A todo lo anteriormente expuesto, se ha de agregar la controversia existente entre el tratamiento del THT a través de un acceso torácico, abdominal o toracoabdominal ${ }^{5,6}$.

El diagnóstico suele realizarse en el preoperatorio por la presencia de manifestaciones clínicas, confirmadas mediante estudios radiológicos, tomográficos y broncoscópicos ${ }^{7}$; sin embargo, en algunos casos puede ser un hallazgo intraoperatorio en pacientes oligosintomáticos.

La cirugía sigue siendo el tratamiento de elección, con una mortalidad quirúrgica que oscila entre $0,9 \%$ y $3,6 \%$ y hasta $9,7 \%$ cuando existe una fístula broncobiliar ${ }^{5,8}$; pronóstico que empeora frente a una segunda y tercera cirugía, situación que incrementa estas cifras entre $6 \%$ y $20 \%$. La técnica quirúrgica a emplear suele depender de la localización de la lesión, del estado del quiste, su tamaño y la experiencia del equipo quirúrgico ${ }^{5}$.

El objetivo de este estudio es describir características clínicas y morbilidad de una serie de pacientes con THT intervenidos quirúrgicamente.

\section{Material y Método}

Diseño del estudio: Serie de casos prospectiva con seguimiento.

Población en estudio: Todos los pacientes con HH que desarrollaron como complicación de su enfermedad hidatídica un THT y que fueron intervenidos quirúrgicamente en el período enero de 1997 a julio de 2007 en el Hospital Regional de Temuco, de forma consecutiva, por el primer autor (CM). El tiempo de seguimiento mínimo fue de 12 meses. No se excluyeron pacientes; por lo que se trata de una muestra no probabilística de casos consecutivos.

Protocolo de estudio: Todos los pacientes fueron estudiados mediante pruebas diagnósticas generales, de función hepática, inmunodiagnóstico, ecotomografía abdominal y radiología de tórax para confirmar enfermedad pulmonar coexistente. Después del alta hospitalaria, los pacientes fueron seguidos con controles clínicos estrictos a lo menos en los meses 1, 6, 12, 24, 36, 48 y 60; oportunidad en las que se realizaba evaluación clínica, de laboratorio general, función hepática, inmunodiagnóstico con determinación de ELISA-IgE, ELISAIgG; y ecotomografía abdominal.

Variables en estudio: La variable resultado fue "desarrollo de morbilidad postoperatoria", medida al menos 12 meses después de la cirugía.

Procedimiento quirúrgico: Las técnicas quirúrgicas empleadas fueron quistectomía subtotal ${ }^{9}$, periquistectomía o lobectomía hepática derecha; frenoplastia y reparación pulmonar según necesidad.

Definiciones: Se definió como THT completo a la afectación del hígado, diafragma y al menos la pleura parietal ${ }^{6,10}$. Las lesiones fueron clasificadas según la propuesta de Gómez R., et $\mathrm{al}^{11}$, en lesiones grados I al V (Figura 1).

Plan de análisis: Se diseñó una hoja computacional de recolección de datos donde se ingresaron las variables clínicas, de laboratorio, de imágenes, de terapia y evolución (desarrollo de complicaciones y mortalidad) de los pacientes; ésta, al 


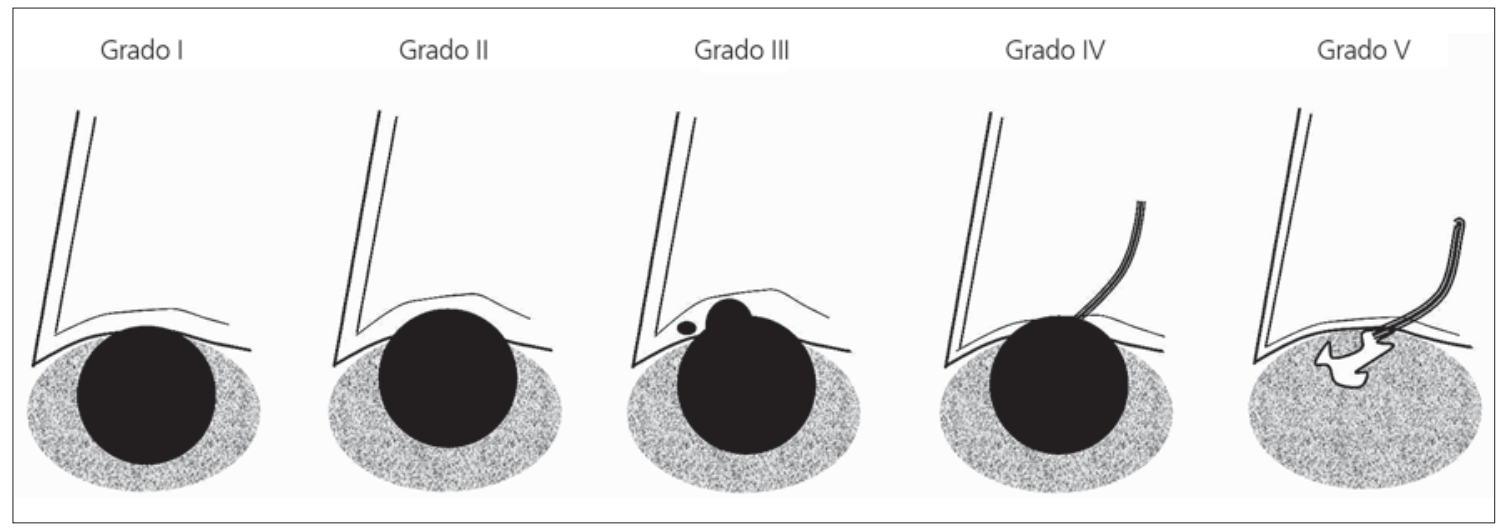

Figura 1. Grado I: Quiste adherido. Existen adherencias firmes entre la superficie del quiste y el diafragma, pero sin perforación de este. Grado II: Tránsito hidatídico. El quiste perfora el diafragma con una pequeña invasión de la cavidad torácica. El defecto diafragmático requiere reparación. Grado III: Vesiculación pleurotorácica. El quiste perfora el diafragma y crece dentro de la cavidad torácica u ocurre un implante de vesículas hijas. Grado IV: Enfermedad del parénquima pulmonar. El quiste se conecta con el árbol bronquial, generándose una fístula quistobronquial; o hay compresión y atelectasia del parénquima pulmonar. Grado V: Fístula bronquial crónica. Estado crónico de la enfermedad en el que existe (n) fístula (s) quistobronquial (es); ya sea secundario a ruptura espontánea del quiste al árbol bronquial o secundario a un postoperatorio.

igual que el tratamiento de los datos, fueron realizados por investigadores independientes (NA, JS, $\mathrm{PU})$. Los datos fueron analizados con el programa Stata 9.0/SE ${ }^{\circledR}$. Se utilizó estadística descriptiva, aplicando medidas de tendencia central y dispersión, con cálculos promedios y desviaciones estándar, medianas y valores extremos; además, se calcularon porcentajes e intervalos de confianza del $95 \%$ (IC 95\%).

\section{Resultados}

En el período estudiado, fueron intervenidos por el primer autor, un total de 243 pacientes por $\mathrm{HH}$; de los cuales, 23 presentaban un THT, lo que representa una incidencia de $0,23 / 100.000$ habitantes por año.

Los pacientes en estudio, con una mediana de edad de 48 años y una distribución por género de $56 \%$ de mujeres; no registraban cirugías previas por $\mathrm{HH}$; sin embargo, 6 de ellos $(26,1 \%)$, presentaban comorbilidad donde destacaba cardiopatía coronaria en 2 casos. Tres pacientes $(13,1 \%)$ presentaban tos y biliptisis.

De los estudios preoperatorios, destaca la alteración de las pruebas hepáticas (Tabla 1 ).

Del estudio de imágenes, destaca el hecho que las lesiones eran únicas en el $65,2 \%$ de los casos, que en su mayoría se localizaron en el lóbulo dere-

Tabla 1. THT. Distribución de las variables contínuas observadas $(\mathbf{n}=\mathbf{2 3})$

\begin{tabular}{lcccc}
\hline Variable & Mediana & Valor mínimo & Valor máximo & IC 95\% \\
\hline Edad (años) & 48 & 16 & 75 & $(42,5-56,9)$ \\
Hematocrito $\left(\mathrm{x} \mathrm{mm}^{3}\right)$ & 36 & 22 & 48 & $(35,0-39,7)$ \\
Leucocitos $(\mathrm{x} \mathrm{mm})^{3}$ & 9.500 & 5.760 & 21.800 & $(9.140,8-12.576,6)$ \\
Bilirrubina (mg/dL) & 0,6 & 0,2 & 3,8 & $(0,5-1,4)$ \\
Fosfatasa alcalina (U/L) & 414 & 69 & 1.052 & $(355,9-617,3)$ \\
ASAT (U/L) & 30 & 3 & 1.044 & $(3,0-179,5)$ \\
ALAT (U/L) & 32 & 6 & 738 & $(6,0-147,5)$ \\
Diámetro del quiste (cm) & 20 & 8 & 30 & $(12,0-25,0)$ \\
Estancia hospitalaria (días) & 5 & 2 & 18 & $(4,6-8,2)$ \\
Seguimiento (meses) & 71 & 12 & 122 & $(46,2-80,8)$ \\
\hline
\end{tabular}

ASAT: Aspartato aminotransferasa. ALAT: Alanino aminotransferasa. 
Tabla 2. THT. Distribución de las variables clínicas observadas $(n=23)$

\begin{tabular}{|c|c|c|}
\hline Variable & n casos & $\%$ \\
\hline \multicolumn{3}{|l|}{ Otras localizaciones } \\
\hline Pulmonar & 2 & 8,7 \\
\hline Peritoneal & 2 & 8,7 \\
\hline Esplénico & 1 & 4,3 \\
\hline \multicolumn{3}{|l|}{ Morbilidad asociada } \\
\hline Cardiopatía coronaria & 2 & 8,7 \\
\hline Diabetes Mellitus & 1 & 4,3 \\
\hline Desnutrición & 1 & 4,3 \\
\hline Embarazo & 1 & 4,3 \\
\hline Colelitiasis & 1 & 4,3 \\
\hline \multicolumn{3}{|l|}{ Hallazgos radiológicos } \\
\hline Normal & 2 & 8,7 \\
\hline Imagen quística & 8 & 34,8 \\
\hline Ascenso diafragmático & 13 & 56,5 \\
\hline \multicolumn{3}{|l|}{ Hallazgos ecográficos } \\
\hline Imagen hipoecoica & 8 & 34,8 \\
\hline Imagen heterogénea & 14 & 60,9 \\
\hline Imagen sólida & 1 & 4,3 \\
\hline \multicolumn{3}{|l|}{ Número de quistes } \\
\hline Único & 15 & 65,2 \\
\hline Dos & 3 & 13,1 \\
\hline Tres o más & 5 & 23,7 \\
\hline \multicolumn{3}{|l|}{ Localización de la lesión principal } \\
\hline Lóbulo derecho & 18 & 78,3 \\
\hline Lóbulo izquierdo & 1 & 4,3 \\
\hline Bilateral & 4 & 17,4 \\
\hline \multicolumn{3}{|l|}{ Comunicaciones biliares } \\
\hline Ninguna & 5 & 21,7 \\
\hline Una & 13 & 56,6 \\
\hline Dos & 5 & 21,7 \\
\hline \multicolumn{3}{|l|}{ Complicaciones evolutivas concomitantes } \\
\hline Absceso hepático de origen hidatídico & 9 & 39,1 \\
\hline Colangiohidatidosis & 1 & 4,3 \\
\hline Ambas & 1 & 4,3 \\
\hline Fístula cistogástrica y anafilaxia & 1 & 4,3 \\
\hline
\end{tabular}

cho $(78,3 \%)$ y que tenían un diámetro cuya mediana era de $20 \mathrm{~cm}$ (Tablas 1 y 2 ).

Respecto del grado de avance del THT, se verificó que 9 pacientes $(39,1 \%)$ presentaban grado I; 7 casos $(30,4 \%)$, grado II; 4 sujetos $(17,4 \%)$, grado III; 3 pacientes $(13,1 \%)$, grado IV; y ningún caso se encontraba en grado $\mathrm{V}$.

Se evidenció concomitancia con otras complicaciones evolutivas de la $\mathrm{HH}$, entre las que destacó la coexistencia de AHH (Tabla 2).

El procedimiento quirúrgico realizado con mayor
Tabla 3. THT. Distribución de las variables quirúrgicas observadas $(n=23)$

\begin{tabular}{lrr}
\hline Variable & n casos & \% \\
\hline Tipo de cirugía realizada sobre el quiste & & \\
$\quad$ Quistectomía subtotal & 18 & 78,3 \\
Periquistectomía & 2 & 8,7 \\
Lobectomía hepática & 3 & 13,0 \\
Tratamiento de la cavidad residual & & \\
Nada & 8 & 34,8 \\
Capitonaje & 7 & 30,4 \\
Epiploplastía & 8 & 34,8 \\
& & \\
Otros procedimientos quirúrgicos realizados & & \\
$\quad$ Ninguno & 5 & 21,7 \\
Colecistectomía & 12 & 52,2 \\
Colecistectomía y coledocostomía & 5 & 21,7 \\
Cirugía de hidatidosis peritoneal & 1 & 4,3 \\
\hline
\end{tabular}

frecuencia sobre la(s) lesión(es) quística(s) fue quistectomía subtotal $(78,3 \%)$; y la cavidad residual se trató preferentemente con epiploplastía $(34,8 \%)$. A esto, se adicionó una frenoplastía con sutura continua de material absorbible en la totalidad de los casos. En ninguno de los casos en que se verificó compromiso pulmonar (3 casos, 13,1\%), se practicó resección parenquimatosa; y el tratamiento de la fístula biliobronquial con el subsecuente cese de los síntomas fue asociado solamente a la eliminación del quiste hepático y el reparo diafragmático; el que se practicó en todos los pacientes con sutura continua de ácido poliglicólico 1. Adicionalmente, se practicó colecistectomía en el 52,2\% de los pacientes (Tabla 3 ).

Se registró una mediana de estancia hospitalaria y de seguimiento de 6,4 días y 71 meses respectivamente (Tabla 1 ). Se verificó un $26,0 \%$ de morbilidad; ausencia de reintervenciones y una mortalidad de 4,4\%. La morbilidad relacionada fue secundaria a: complicaciones pulmonares ( 5 casos; $21,7 \%$ ) e infección del sitio operatorio ( 1 caso; 4,3\%). La muerte observada fue secundaria a síndrome de distress respiratorio agudo postoperatorio en una paciente que evolucionó con una neumonía.

\section{Discusión}

Existen pocos estudios referentes a THT, la mayor parte de los cuales corresponden a series de casos de carácter retrospectivo, de pocos pacientes; o, en los que se alude al tema de forma colate$\mathrm{ral}^{10}$. Aún así, la incidencia encontrada en este estudio es similar a otras previamente reportadas ${ }^{1,11}$. 
Concordante con la literatura, la mayor frecuencia de localización fue el lóbulo hepático derecho ${ }^{12}$ y llama la atención que la mediana del diámetro corresponde a quistes de gran tamaño $(20 \mathrm{~cm})$.

Otro hecho que parece de interés discutir es la baja frecuencia de fístula biliobronquial; entidad poco frecuente, de la que suelen encontrarse sólo reportes aislados de $\operatorname{casos}^{13-15}$; sin embargo, en otras series, se reporta una prevalencia de esta complicación en pacientes con $\mathrm{HH}$ complicada de $3,5 \%{ }^{16}$, y en pacientes con THT de 5,3\% ${ }^{11}$.

Aunque la vía de acceso es controversial, se utilizó la vía abdominal debido a la alta prevalencia de complicaciones evolutivas de la $\mathrm{HH}$ concomitantes al THT (absceso hepático de origen hidatídico [ 9 casos; $39,1 \%$ ], colangiohidatidosis [ 1 caso; $4,3 \%$ ], fístula cistogástrica y anafilaxia [1 caso; $4,3 \%]$ ). Para ello se utilizó una laparotomía en J; pues por una parte, el acceso que se obtiene con ella es muy apropiado para el adecuado tratamiento de pacientes con patología hepatobiliar; y por otra, es una laparotomía que no tiene mayor morbilidad que la que se observa con otro tipo de accesos (laparotomía subcostal, laparotomía media) ${ }^{17}$.

En la literatura, destaca una morbilidad quirúrgica para este tipo de pacientes de $14,0 \%$ a $35,0 \%$; con una tasa de ISO de $17,5 \%$. Y una mortalidad de $7,5 \%$ a $8,9 \%^{1,18}$; cifras superiores a las observadas en esta serie. Dispersión que puede explicarse por variables como lo heterogéneo de las series publicadas, el tipo de acceso utilizado y la indicación de resección pulmonar concomitante en casos de afectación parenquimatosa con fístula biliobronquial.

\section{Referencias}

1. Kilani T, Hammami S, Horchani H, Ben Miled-Mrad K, Hantous S, Mestiri I, et al. Hydatid disease of the liver with thoracic involvement. World J Surg 2001; 25: $40-45$.

2. Manterola C, Barroso M, Vial M, Bustos L, Muñoz S, Losada $\mathrm{H}$, et al. Liver abscess of hydatid origin: clinical features and results of aggressive treatment. ANZ J Surg 2003; 73: 220-224.

3. Manterola C, Losada H, Carrasco R, Muñoz S, Bustos L, Vial M, y col. Colangiohidatidosis. Una complicación evolutiva de la hidatidosis hepática. Bol Chil Parasitol 2001; 56: 10-15.

4. Manterola C, Vial M, Pineda V, Sanhueza A, Barroso M. Factors associated with morbidity in liver hydatid surgery. ANZ J Surg 2005; 75: 889-892.

5. Tocchi A, Mazzoni G, Miccini M, Drumo A, Cassini D, Colace L, et al. Treatment of hydatid broncho- biliary fistulas: 30 years experience. Liver Int 2007; 27: 209-214.

6. Kilani T, Hammami S, Horchani H, Ben Miled-Mrad $\mathrm{K}$, Hantous S, Mestiri I, et al. Hydatid disease of the liver with thoracic involvement. World J Surg 2001; 25: 40-45.

7. Uzun K, Ozbay B, Etlik O, Kotan C, Gencer M, Sakarya ME. Bronchobiliary fistula due to hydatid disease of the liver: a case report. Acta Chir Belg 2002; 102: 207-209.

8. Gerazounis M, Athanassiadi K, Metaxas E, Athanassiou M, Kalantzi N. Bronchobiliary fistulae due to echinococcosis. Eur J Cardiothorac Surg 2002; 22: 306-308.

9. Manterola C, Molina E, Fernández O, Barroso M. Quistectomía subtotal. Una alternativa quirúrgica racional en el tratamiento de la hidatidosis hepática. Rev Chil Cir 1998; 50: 621-629.

10. Manterola Delgado C, Barroso Vásquez M, Oberg Aravena C, Molina Villarroel E, Vial Gallardo M, Fernández Arancibia O. Alternativas quirúrgicas en el tratamiento de la hidatidosis. Bol Chil Parasitol 1999; 54: $13-20$

11. Gómez R, Moreno E, Loinaz C, De la Calle A, Castellón C, Manzanera M, et al. Diaphragmatic or transdiaphragmatic thoracic involvement in hepatic hydatid disease: surgical trends and classification. World J Surg 1995; 19: 714-719.

12. Boyd DP. Bronchobiliary and bronchopleural fistulas. Ann Thorac Surg 1977; 24: 481-487.

13. Kim YS, Rhim H, Sung JH, Kim SK, Kim Y, Koh BH, et al. Bronchobiliary Fistula after Radiofrequency Thermal Ablation of Hepatic Tumor. J Vasc Intervent Radiol 2005; 16: 407-410.

14. Rodrígues OR, Quim ACO, Minamoto H, Matheus RS, Schmidt Jr AF. Fístula bílio-bronquica: relato de caso e revisao da literatura. Acta Cir Bras 1998; 13: $264-$ 268.

15. Losada H, Vial M, Manterola C, Pineda V. Fístula biliobronquial secundaria a quiste hepático hidatídico en tránsito al tórax. Reporte de caso. Rev Chil Cir 2006; 58: 224-227.

16. Chautems R, Buhler LH, Gold B, Giostra E, Poletti $\mathrm{P}$, Chilcott M, et al. Surgical management and longterm outcome of complicated liver hydatid cyst caused by Echinococcus granulosus. Surgery 2005; 137: 326328.

17. Manterola C, Muñoz S, Fernández O, Molina E, Barroso M. Laparotomía en jota. Una vía de acceso opcional para cirugía abdominal alta. Rev Chil Cir 1999; 51: 275-282.

18. Kabiri EH, El Maslout A, Benosman A. Thoracic rupture of hepatic hydatidosis (123 cases). Ann Thorac Surg 2001; 72: 1883-1886. 